\title{
RANKL reverse signalling and bone
}

New research by Hiroshi Suzuki and colleagues show that reverse signalling of receptor activator of nuclear factor- $\kappa \mathrm{B}$ (RANK; also known as TNFRSF11A) ligand (RANKL; also known as TNFSF11) in osteoblasts has a role in linking bone resorption and formation.

RANKL belongs to the tumour necrosis factor family, which is a group of proteins that act as bidirectional signalling molecules and produce intracellular reverse signalling. Previously, osteoblasts were thought to be the main producers of RANKL; however, mounting evidence suggests that osteocytes are the main source during bone remodelling. "Because of this finding, we hypothesized that osteoblastic RANKL might have a different physiological role than that of osteocytic RANKL," explains corresponding author Masashi Honma.

In this study, the researchers showed that maturing osteoclasts secreted vesicular RANK (vRANK), which binds to osteoblastic RANKL and activates reverse signalling through Runx2. The stimulation also increased the mineralization of osteoblasts.
Next, the authors created a mouse model $\left(\right.$ Rankl $\left.{ }^{P 29 A}\right)$ to suppress reverse signalling of RANKL but not forward signalling, which triggers osteoclastogenesis. When recombinant RANKL was administered, osteoclast maturation in both $\operatorname{Rank}^{P 29 A}$ and wild-type mice was increased; however, bone formation was disrupted in $\operatorname{Rankl}^{P 29 A}$ mice compared with wild-type mice. The results suggest that osteoblastic RANKL reverse signalling is involved in linking bone resorption and formation.

Finally, Suzuki and colleagues targeted RANKL reverse signalling in an ovariectomized mouse model to test whether it is a possible pharmacological target for the treatment of osteoporosis. They found that activation of RANKL reverse signalling inhibited reduced bone formation. "Our findings indicate that the role of RANKL is the accelerator of bone turnover rather than the stimulator of bone resorption," concludes Honma.

Ivone Leong

ORIGINAL ARTICLE Ikebuchi, Y. et al. Coupling of bone resorption and formation by RANKL reverse signalling. Nature https://doi.org/10.1038/s41586-018-0482-7 (2018)

\section{GENETICS}

\section{Shared variants for osteoporosis and T2DM}

Epidemiological evidence has suggested that there is an association between osteoporosis and type 2 diabetes mellitus (T2DM); however, the mechanisms underlying this association have been unclear. Now, new research has identified genetic variants that could account for the link between osteoporosis and T2DM.

"An important task and challenge is to perform a systematic search for shared genetic loci influencing these two diseases," explains Hong-Wen Deng. "We applied the pleiotropic conditional false discovery rate (cFDR) method to two sets of data from genome-wide association studies to identify novel variants with pleiotropic effects on femoral BMD and T2DM." The analysis included 53,236 participants with data on femoral neck BMD and 159,208 participants with data on T2DM.

The researchers identified 27 SNPs associated with femoral neck BMD and 61 SNPs associated with T2DM. Of these SNPs, four were shared between femoral neck BMD and T2DM, indicating that they could be pleiotropic. One of these SNPs was located in PLEKHA1; further analysis revealed that this gene is differentially expressed between patients with high versus low BMD and between patients with or without T2DM. Weighted gene co-expression analyses also showed that PLEKHA1 is associated with multiple genes that are known to be involved in osteoporosis or T2DM.

"Our study highlights PLEKHA1 as an important potentially pleiotropic gene," says Deng. PLEKHA1 is involved in regulating phosphorylation, which in turn regulates a range of cellular processes, including insulin sensitivity and bone mineralization. "Therefore, PLEKHA1 might participate in bone and T2DM metabolism," concludes Deng. The researchers hope that future studies will build on these findings, including exploring the role of PLEKHA1 in osteoporosis and T2DM in more detail. The authors also note that their findings need to be validated.

Claire Greenhill

ORIGINAL ARTICLE Hu, Y. et al. Identification of novel variants associated with osteoporosis, type 2 diabetes and potentially pleiotropic loci using pleiotropic cFDR method. Bone https://doi.org/10.1016/j.bone.2018.08.020 (2018)

\section{Role of PKC $\varepsilon$ in insulin resistance}

Increased levels of diacylglycerol (DAG) in the liver are associated with hepatic insulin resistance. Now, new research by Brandon Gassaway, Varman Samuel, Gerald Shulman and Jesse Rinehart reports that protein kinase $C_{\varepsilon}(\mathrm{PKC} \varepsilon)$ is a key contributor in a network of proteins that might drive hepatic insulin resistance, particularly by interacting with $70 \mathrm{kDa}$ ribosomal protein $\mathrm{S} 6$ kinase (P70S6K), a key kinase in the insulin signalling pathway.

Previous studies in humans and rodents showed that increased levels of DAG activate PKC $\varepsilon$, which then impairs the insulin receptor kinase activity. "As PKC activity has been connected to insulin resistance for decades, we hypothesized that a DAG-activated PKC $\varepsilon$ would target a network of proteins and that some of these targets might contribute to the complex phenotype that is insulin resistance," explains Rinehart.

To study the signalling pathways affected by $P K C \varepsilon$, the researchers fed two groups of rats a high-fat diet (HFD): normal rats and rats with $\mathrm{PKC} \varepsilon$ knocked down. Liver samples were taken and analysed using phosphoproteomic methods to compare sites of protein phosphorylation between the two groups. Surprisingly, many of the proteins affected by PKC $\varepsilon$ had not previously been associated with insulin signalling.

Next, the researchers tested a selection of candidate proteins with a small interference RNA-based screen to identify which proteins have novel interactions with insulin signalling. The authors showed that many of the proteins did regulate insulin signalling and that $\mathrm{PKC} \varepsilon$ itself was an activator of the insulin signalling pathway. Other novel interactors with insulin signalling included $40 \mathrm{~S}$ ribosomal protein S6 (RPS6) and neogenin 1 (NEO1).

Finally, Rinehart and colleagues found that IRS1 and RPS 6 are also substrates of PKC $\varepsilon$ and suggested that $\mathrm{PKC} \varepsilon$ crosstalked with $\mathrm{P} 70 \mathrm{~S} 6 \mathrm{~K}$ via the two kinases. The authors concluded that this crosstalk is involved in HFD-induced insulin resistance. "We now need to understand how each of these PKC $\varepsilon$ targets might contribute to hepatic insulin resistance," concludes Samuel.

Ivone Leong

ORIGINAL ARTICLE Gassaway, B. M. et al. PKC $\varepsilon$ contributes to lipid-induced insulin resistance through cross talk with p70S6K and through previously unknown regulators of insulin signaling. Proc. Natl Acad. Sci. USA https://doi. org/10.1073/pnas.1804379115 (2018) 